\title{
Symbolic Determination of Jacobian and Hessian Matrices and Sensitivities of Active Linear Networks by Using Chan-Mai Signal-Flow Graphs
}

\author{
Georgi A. Nenov \\ Higher School of Transport "T. Kableshkov", Sofia, \\ Bulgaria
}

\section{Introduction}

Every network synthesis procedure normally includes a first-order or (more rarely) secondorder network sensitivity analysis. The main problem here is the evaluation of the corresponding first- or second-order derivatives of network functions with respect to the circuit element values. These derivatives form the network Jacobian $(\mathbf{J})$ and Hessian $(\mathbf{H})$ matrices, respectively. A variety of methods exist for such an evaluation but most of them are intended for the sensitivity of one network transfer function only. Besides this in many cases it is desirable to find the symbolic expressions of the sensitivities because such a presentation facilitates the element value influence determination. An other useful and important application of the matrices $\mathbf{J}$ and $\mathbf{H}$ is in the tasks for optimization of synthesized networks with respect to their sensitivities or other parameters (Korn \& Korn, 1968; Wilde,1978).

As it is well known all linear active networks can be modeled by using passive elements and nullator-norator pairs (nullors). The presented paper deals with the application of Chan-Mai signal-flow graphs (CMG) to the determination of the matrices $\mathbf{J}$ and $\mathbf{H}$ elements, having in mind the peculiarities of nullors and their influence on the passive element network admittance matrix and on the corresponding CMG. The method developed here is an improved and enlarged version of the approach in (Nenov, 2004). One demonstrates that the method reduces to the obtaining of two (for the elements of $\mathbf{J}$ ) or four (for the elements of $\mathbf{H}$ ) isomorphic Chan-Mai signal-flow graphs.

\section{Chan-Mai signal flow graph}

It was introduced in graph theory in 1967 (Chan \& Mai, 1967). Compared with other kinds of oriented graphs (especially Mason and Coates graphs) the Chan-Mai graph (CMG) holds out a simplest way to the representation the relationships between the dependent and independent quantities in an algebraic equation set. In order to make easier the understanding of the following sections of the paper further we give the procedure for drawing of CMG and the basic formulae related. 
Assume the algebraic set

$$
\text { A.X }=\mathbf{Y}
$$

is given, where

$$
\mathbf{A}=\left[\begin{array}{cccc}
a_{11} & a_{12} & \ldots & a_{1 n} \\
a_{21} & a_{22} & \ldots & a_{2 n} \\
\cdot & \cdot & \cdot & \cdot \\
a_{n 1} & a_{n 2} & \cdot & a_{n n}
\end{array}\right]
$$

is a square matrix with real or complex entries and

$$
\left.\begin{array}{l}
\mathbf{X}=\left[\begin{array}{llll}
x_{1} & x_{2} & \ldots & x_{n}
\end{array}\right]_{t} ; \\
\mathbf{Y}=\left[\begin{array}{llll}
y_{1} & y_{2} & \ldots & y_{n}
\end{array}\right]_{t}
\end{array}\right\}
$$

are the vectors of the dependent and of the independent variables, respectively. The CMG consists of $n$ vertices with sink signals $y_{1}, y_{2}, \ldots, y_{n}, n$ vertices with source signals $x_{1}, x_{2}, \ldots, x_{n}$ and maximum $n^{2}$ edges with transmission coefficients $a_{j i}$ directed from the vertex $x_{i}$ toward the vertex $y_{j} ; i, j=1,2, \ldots, n$ - Fig. 1 . The calculations on the base of a CMG are connected with the following definitions (Chan \& Mai, 1967, Donevsky \& Nenov, 1979):

i. By removing all outgoing from the vertex $x_{i}$ edges and by adding the edges with transmission coefficients $y_{j}$ from the vertex $x_{i}$ directed toward the vertices $y_{j}, j=1,2, \ldots$, $n$ one obtains the graph $\mathrm{CMG}, i$;

ii. A separation (S) contains all vertices of CMG and a part of edges so that every vertex is incident to only one incoming and one only outgoing edge. The product of the transmission coefficients of all edges in a separations represents the corresponding separation product (SP);

iii. Two edges with transmission coefficients $a_{i j}$ and $a_{j i}$ form a symmetrical pair.

iv. An edge which does not belong to a symmetrical pair is an asymmetrical edge.

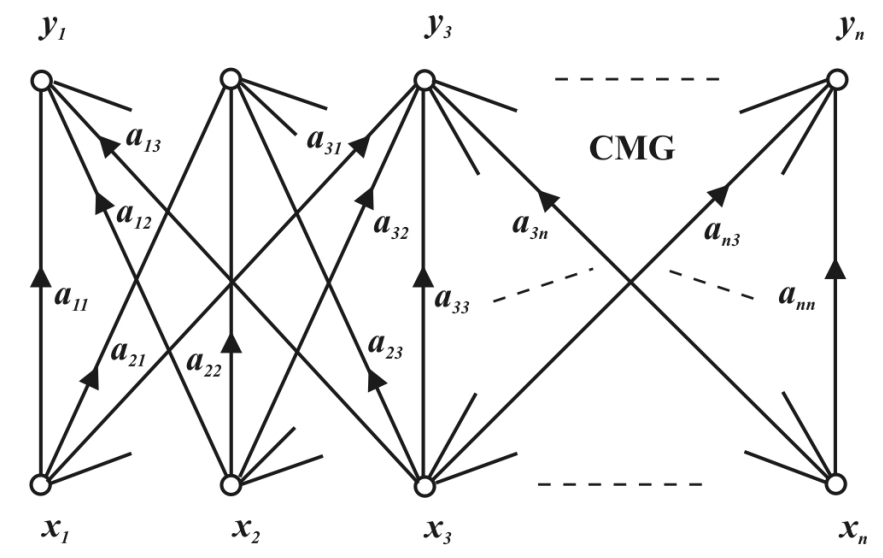

Fig. 1. Chan-Mai Signal-Flow Graph 
An arbitrary unknown quantity $x_{i}$ in $\mathbf{X}$ can be evaluated according to the expression

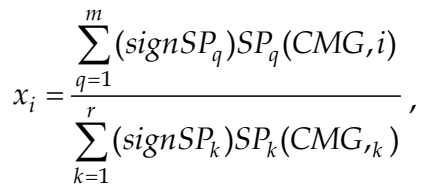

where

$$
\begin{aligned}
& \operatorname{signSP}_{l}= \begin{cases}(-1)^{N_{s, l}+N_{a, l}-1} & \text { for } N_{a, l} \neq 0 \\
(-1)^{N_{s, l}} & \text { for } N_{a, l}=0\end{cases} \\
& l=q \text { or } k
\end{aligned}
$$

In (4) and (5) $r$ is the number of the separations in CMG, $m$ is the number of the separations in $\mathrm{CMG}_{, i}, N_{a, k}$ is the number of all asymmetrical edges in $k$-th separation of CMG, $N_{s, k}$ is the number of all symmetrical pairs in $k$-th separation of CMG, $N_{a q}$ is the number of the asymmetrical edges in $q$-th separation of $\mathrm{CMG}, i, N_{s, q}$ is the number of the symmetrical pairs in $q$-th separation of $C M G, i$, whereas $S P_{q}(C M G, i)$ and $S P_{k}(C M G)$ are the separation products of $q$-th separation of $\mathrm{CMG}, i$ and the separation products of $k$-th separation of $\mathrm{CMG}$, respectively.

\section{Nullor network Chan-Mai signal-flow graph}

Suppose that an equivalent nullor network $N$ with $m+1$ nodes, $r$ passive branches and $g$ nullors is given and the nodal equation of its passive part $N_{\mathrm{p}}$ (the part of $N$ which is obtained by removing all nullors) is

$$
\mathbf{Y}_{p} \mathbf{V}_{p}=\mathbf{I}_{p}
$$

where

$$
\mathbf{Y}=\left[\begin{array}{ccc}
Y_{p, 11} & \ldots & Y_{p, 1 m} \\
\cdot & \cdot & \cdot \\
Y_{p, m 1} & \ldots & Y_{p, m m}
\end{array}\right]
$$

is the nodal matrix of $N_{\mathrm{p}}$ and

$$
\left.\begin{array}{l}
\mathbf{V}_{p}=\left[\begin{array}{llll}
V_{p, 1} & V_{p, 2} & \ldots & V_{p, m}
\end{array}\right]_{t} ; \\
\mathbf{I}_{p}=\left[\begin{array}{llll}
I_{p, 1} & I_{p, 2} & \ldots & I_{p, m}
\end{array}\right]_{t}
\end{array}\right\}
$$

are the nodal voltage and the nodal current vectors of $N_{p}$, respectively. Additionally we assume that between the nodes of all node pairs in $N$ only one element or more than one but parallel connected elements exist. 
The equation (1) can be represented graphically by using a CMG $G_{\mathrm{p}}$ (Chan \& Mai, 1967). Further, taking into account the peculiarities of the nullators and the norators (Davies, 1966) the graph $G_{\mathrm{p}}$ can be transformed into the graph $G$ of the actual network $N$ according to the following

Rule 1:

i. When a nullator is connected between the node $k$ in $N$ and the ground node $m+1$ one removes all vertices going out from the vertex $V_{k}$ of $G_{\mathrm{p}}$;

ii. When a norator is connected between the node $k$ in $N$ and the ground node $m+1$ one removes all vertices coming into the vertex $I_{k}$ of $G_{\mathrm{p}}$;

iii. When a nullator is connected between the nodes $k$ and $l$ in $N$ one unites the vertices $V_{k}$ and $V_{l}$ in $G_{p}$;

iv. When a norator is connected between the nodes $k$ and $l$ in $N$ one unites the vertices $I_{k}$ and $I_{l}$ in $G_{\mathrm{p}}$.

The so obtained graph CMG G corresponds to the matrix equation

$$
\mathbf{Y V}=\mathbf{I}
$$

where $\mathbf{Y}$ is an $(n \times n)$ nodal admittance matrix of $N, \mathbf{V}$ is the nodal voltage vector of $N$ and $\mathbf{I}$ is the nodal current vector of for $n=m-g$.

\section{Jacobian matrix determination}

The matrices in (9) have the form:

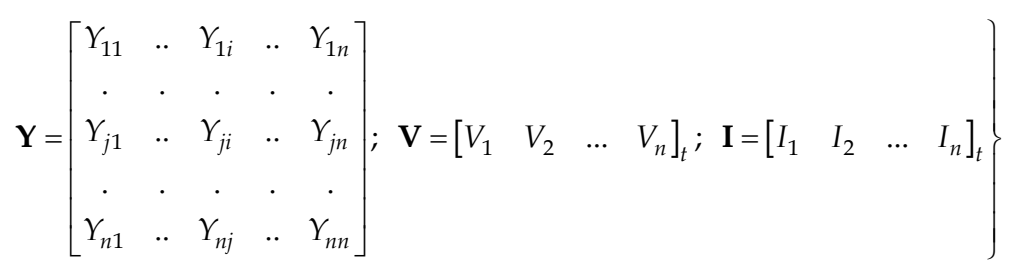

In the common case every element $Y_{j i}$ in (7) is an algebraic admittance sum

$$
Y_{j i}=\sum_{s} y_{s} ; j, i \in\{1,2, \ldots, n\} ; s \in\{1,2, \ldots, r\},
$$

where $y_{s}$ is the admittance of $s$-th branch of the network $N_{p}$.

The vectors $\mathbf{V}$ and $\mathbf{I}$ correspond to the unknown (dependent) variables and to independent variables of $N$, respectively and consequently

$$
\mathbf{V}=\mathbf{Y}^{-1} \mathbf{I}
$$

Let us suppose that the admittance $y_{s}$ changes its value to

$$
y_{s}^{\prime}=y_{s}+d y_{s} \text {. }
$$


Usually the admittance $y_{s}$ takes part in several (but no more then four) elements of (7) and then all these elements change their values (Nenov, 2004)

$$
\left.\begin{array}{c}
Y_{j i}^{\prime}=Y_{j i}+d Y_{j i}=Y_{j i}+\frac{\partial Y_{j i}}{\partial y_{s}} d y_{s} ; \\
j, i \in\{1,2, \ldots, n\} ; s \in\{1,2, \ldots, r\}
\end{array}\right\}
$$

and

$$
\mathbf{Y}^{\prime}=\mathbf{Y}+d \mathbf{Y}
$$

In a common case the admittance $y_{s}$ influences the admittances $Y_{j i}, Y_{j l}, Y_{k i}$ and $Y_{k l} ; i, j, k, l \in\{1$, $2, \ldots, n\}$. Then one obtains

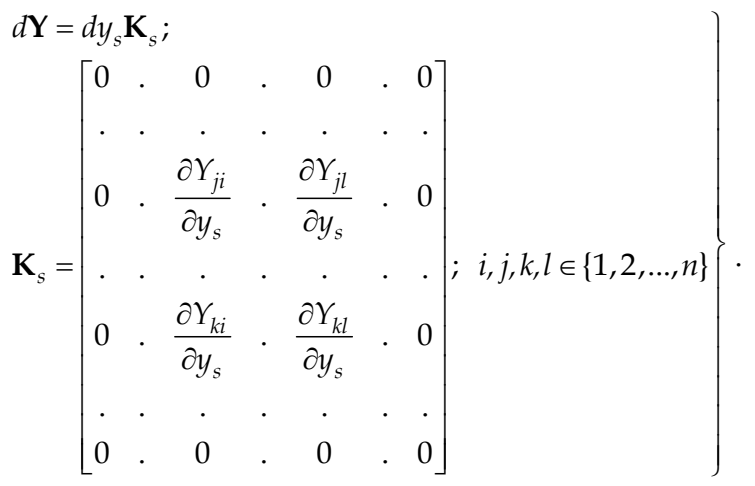

Note that the values of the derivatives in (16) are 1 or -1 because every admittance $y_{s}$ takes part in (11) only once. Hence

$$
\mathbf{V}^{\prime}=\mathbf{V}+d \mathbf{V}
$$

for

$$
d \mathbf{V}=\left(\frac{\partial \mathbf{V}}{\partial Y_{j i}} \frac{\partial Y_{j i}}{\partial y_{s}}+\frac{\partial \mathbf{V}}{\partial Y_{j l}} \frac{\partial Y_{j l}}{\partial y_{s}}+\frac{\partial \mathbf{V}}{\partial Y_{k i}} \frac{\partial Y_{k i}}{\partial y_{s}}+\frac{\partial \mathbf{V}}{\partial Y_{k l}} \frac{\partial Y_{k l}}{\partial y_{s}}\right) d y_{s},
$$

or:

$$
\left.\begin{array}{l}
d \mathbf{V}=d y_{s} \sum_{p q} \frac{\partial \mathbf{V}}{\partial Y_{p q}} \frac{\partial Y_{p q}}{\partial y_{s}} \\
p, q \in\{1,2, \ldots, n\} .
\end{array}\right\}
$$

By substituting $\mathbf{Y}^{\prime}$ and $\mathbf{V}^{\prime}$ in (9) instead $\mathbf{Y}$ and $\mathbf{V}$, respectively, it follows

$$
[\mathbf{Y}+d \mathbf{Y}] \cdot[\mathbf{V}+d \mathbf{V}]=\mathbf{I}
$$


Having in mind that

$$
d \mathbf{Y} d \mathbf{V} \rightarrow \mathbf{0}
$$

the equation (20) yields

$$
\mathbf{Y} d \mathbf{V}=-d \mathbf{Y} \mathbf{V}
$$

or

$$
d \mathbf{V}=-\mathbf{Y}^{-\mathbf{1}} d \mathbf{Y} \mathbf{V}
$$

Then we obtain

$$
\frac{\partial \mathbf{V}}{\partial y_{s}} d y_{s}=-d y_{s} \cdot \mathbf{Y}^{-1} \mathbf{K}_{s} \mathbf{V}
$$

and the Jacobian matrix (Korn \& Korn, 1968). for the change of the admittance $y_{s}$ is

$$
\mathbf{J}=\left[\begin{array}{cccccc}
\frac{\partial V_{1}}{\partial y_{1}} & \frac{\partial V_{1}}{\partial y_{2}} & . . & \frac{\partial V_{1}}{\partial y_{s}} & . . & \frac{\partial V_{1}}{\partial y_{r}} \\
\frac{\partial V_{2}}{\partial y_{1}} & \frac{\partial V_{2}}{\partial y_{2}} & . . & \frac{\partial V_{2}}{\partial y_{s}} & . . & \frac{\partial V_{2}}{\partial y_{r}} \\
\cdot & \cdot & . . & . & . . & . \\
\frac{\partial V_{n}}{\partial y_{1}} & \frac{\partial V_{n}}{\partial y_{2}} & . . & \frac{\partial V_{n}}{\partial y_{s}} & . . & \frac{\partial V_{n}}{\partial y_{r}}
\end{array}\right]=\left[\begin{array}{llllll}
\mathbf{J}_{1} & \mathbf{J}_{2} & . . & \mathbf{J}_{s} & . . & \mathbf{J}_{r}
\end{array}\right]
$$

where

$$
\mathbf{J}_{s}=\left[\begin{array}{llll}
\frac{\partial V_{1}}{\partial y_{s}} & \frac{\partial V_{2}}{\partial y_{s}} & . . & \frac{\partial V_{n}}{\partial y_{s}}
\end{array}\right]_{t} .
$$

Taking into account (24) and (25) one obtains

$$
\left.\begin{array}{l}
\mathbf{J}_{s}=-\mathbf{Y}^{-1} \mathbf{K}_{s} \mathbf{Y}^{-1} \mathbf{I}=-\mathbf{Y}^{-1} \mathbf{K}_{s} \mathbf{V}=-\mathbf{Y}^{-1} \mathbf{V}_{s} ; \\
\mathbf{V}_{s}=\mathbf{K}_{s} \mathbf{V} .
\end{array}\right\}
$$

and according to $(20) \div(22)$

$$
\mathbf{J}=-\mathbf{Y}^{-1}\left[\begin{array}{lllll}
\mathbf{K}_{1} & . . & \mathbf{K}_{s} & . . & \mathbf{K}_{r}
\end{array}\right] \mathbf{V} .
$$

The expressions (22) show that in order to find the vector $\mathbf{J}_{s}$ it is necessary to follow the Rule 2:

i. Find the vector $\mathbf{V}$ by using the CMG $G$;

ii. Evaluate the vector $\mathbf{V}_{s}$;

iii. Draw a new $C M G G_{s}$ where the source vertices are the elements of the vector $\mathbf{J}_{s}$ and the sink vertices are the elements of the vector $\mathbf{V}_{s}$;

iv. Find the source vertex variables in $G_{s}$. 


\section{Example A}

The network $N$ in Fig. 2 is given, where $m=6 ; r=9 ; g=2$. Here obviously $V_{2}=V_{3}=V_{23} ; V_{6}=0$ and we wish to find the vector

$$
\mathbf{J}_{3}=\left[\begin{array}{llll}
\frac{\partial V_{1}}{\partial\left(s C_{3}\right)} & \frac{\partial V_{23}}{\partial\left(s C_{3}\right)} & \frac{\partial V_{4}}{\partial\left(s C_{3}\right)} & \frac{\partial V_{5}}{\partial\left(s C_{3}\right)}
\end{array}\right]_{t} .
$$

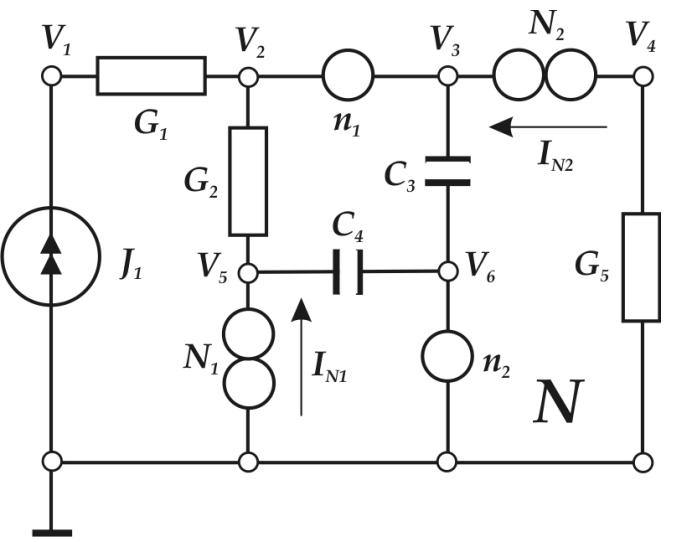

Fig. 2. Nullor Network $N$

In Fig. 3 the $C M G G_{p}$ of the passive part of $N$ is drawn (Nenov, 2004). Further following the Rule 2 we reach to the graph $G$ in Fig. 4 for

$$
\left.\begin{array}{l}
\mathbf{Y}=\left[\begin{array}{cccc}
G_{1} & -G_{1} & 0 & 0 \\
-G_{1} & G_{1}+G_{2} & 0 & -G_{2} \\
0 & s C_{3} & G_{5} & 0 \\
0 & -s C_{3} & 0 & -s C_{4}
\end{array}\right] ; \\
\mathbf{V}=\left[\begin{array}{lllll}
V_{1} & V_{2}=V_{3}=V_{23} & V_{4} & V_{5}
\end{array}\right]_{t} ; \mathbf{I}=\left[\begin{array}{llll}
J_{1} & 0 & 0 & 0
\end{array}\right]_{t}
\end{array}\right\}
$$

Because $Y_{32}={ }_{s} C_{3} ; Y_{42}=-s C_{3}$ and

$$
\frac{\partial Y_{32}}{\partial\left(s C_{3}\right)}=1 ; \frac{\partial Y_{32}}{\partial\left(s C_{3}\right)}=-1
$$

from (16) and (31) we have

$$
\left.\begin{array}{rl}
\mathbf{K}_{3} & =\left[\begin{array}{cccc}
0 & 0 & 0 & 0 \\
0 & 0 & 0 & 0 \\
0 & 1 & 0 & 0 \\
0 & -1 & 0 & 0
\end{array}\right] ; \\
\mathbf{V}_{3} & =\mathbf{K}_{3} \mathbf{V}=\left[\begin{array}{llll}
0 & 0 & V_{23} & -V_{23}
\end{array}\right]_{t}
\end{array}\right\}
$$




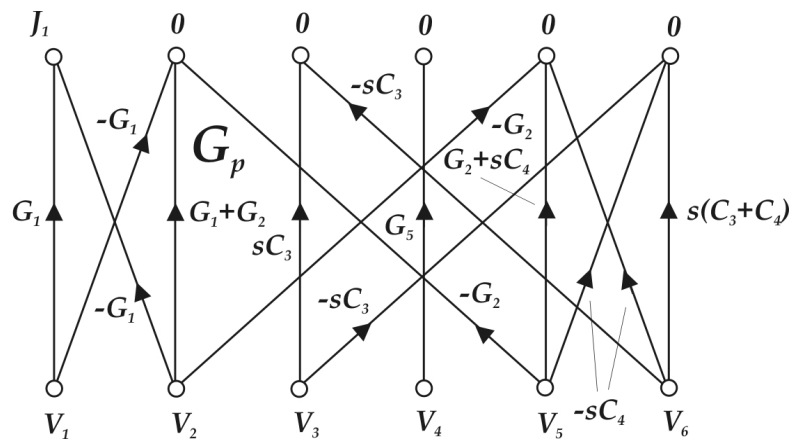

Fig. 3. CM Signal-Flow Graph $G_{p}$

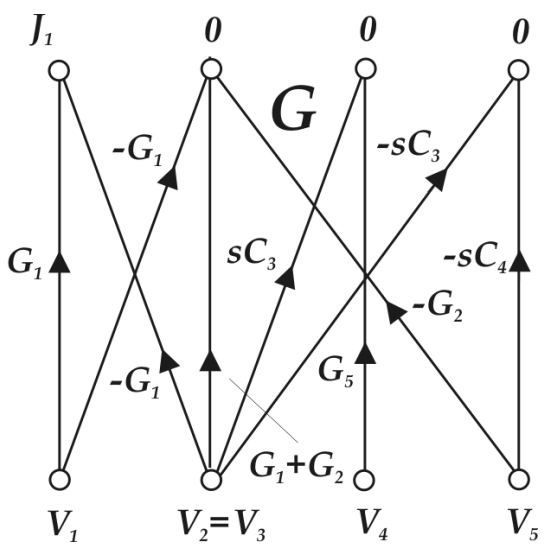

Fig. 4. CM Signal-Flow Graph $G$

Obviously, in the case we have to find the voltage $V_{23}$ only. For this purpose a CM graph $G_{23}$ is drawn (Fig. 5).

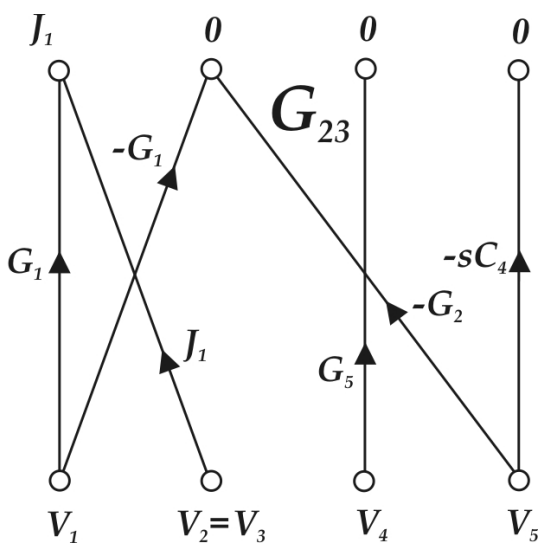

Fig. 5. CM Signal-Flow Graph $G_{23}$ 
According to (4) and (5) for the separations of the graph $G$ (Fig. 6) we obtain

$$
\left.\begin{array}{l}
S P_{1}=-G_{1} s C_{4} G_{5}\left(G_{1}+G_{2}\right) ; N_{a, 1}=4 ; N_{s, 1}=0 ; \\
S P_{2}=-G_{1}^{2} s C_{4} G_{5} ; N_{a, 2}=2 ; N_{s, 2}=1 ; \\
S P_{3}=G_{2} s C_{3} G_{5}: N_{a, 3}=2 ; N_{s, 3}=1
\end{array}\right\}
$$

and for the unique separation of the graph $G_{23}$ (Fig. 7):

$$
S P_{23,1}=J_{1} G_{1} s C_{4} G_{5} ; N_{a, 23,1}=2 ; N_{s, 23,1}
$$

Then the formulae (4) and (5) yield

$$
V_{23}=\frac{J_{1} C_{4}}{G_{2}\left(C_{3}+C_{4}\right)}
$$

Having in mind $(26) \div(29)$ we have
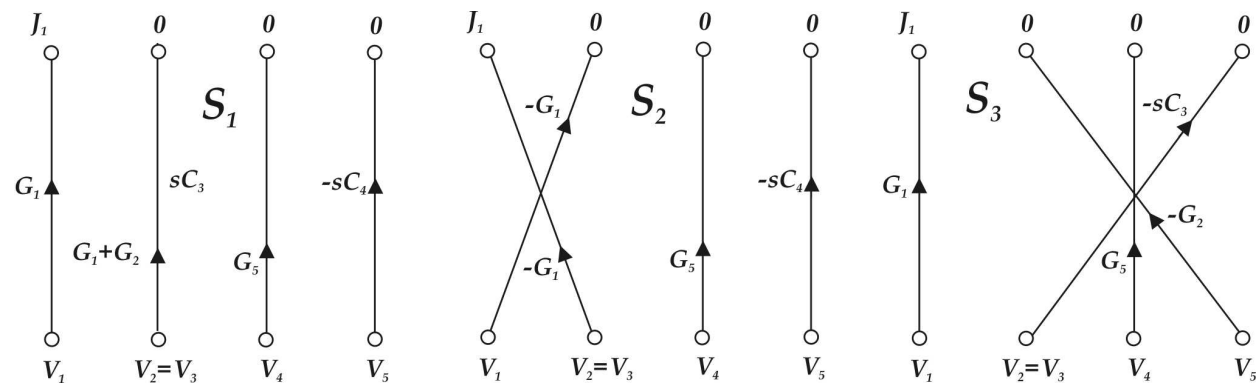

Fig. 6. Separations $S_{1}, S_{2}$ and $S_{3}$ of CM Graph $G$

$$
\mathbf{J}_{3}=-\mathbf{Y}^{-1} \mathbf{V}_{3}=\mathbf{Y}^{-1}\left(-\mathbf{V}_{3}\right)
$$

and following Rule 2 one draws the $\mathrm{CM}$ graph $G_{/ 3}$ (Fig. 8). Obviously, the graphs $G$ and $G_{J 3}$ have one and the same structure and consequently the expressions (33) hold for the source vertex quantities in (29) also. But for the nominator polynomials in (4) we have to draw according the Rule 2 four new CM graphs $-G_{J 3,1}, G_{J 3,23}, G_{J 3,4}$ and $G_{J 3,5}-$ Fig. 9.

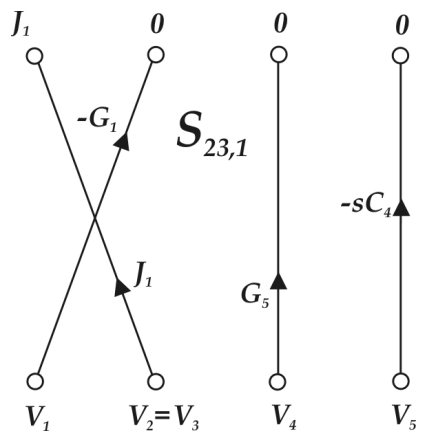

Fig. 7. Separation $S_{23,1}$ of CM Graph $G_{23}$ 


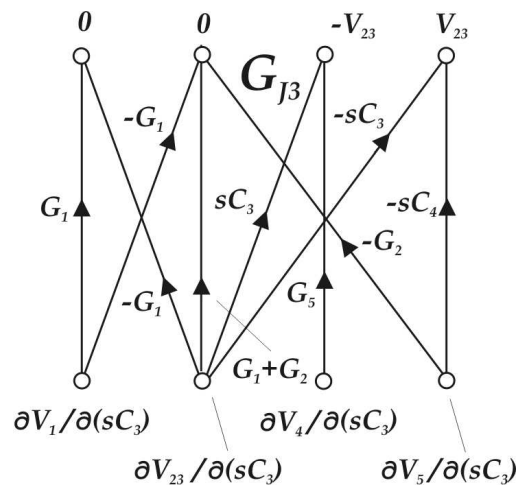

Fig. 8. CM Graph $G_{J 3}$

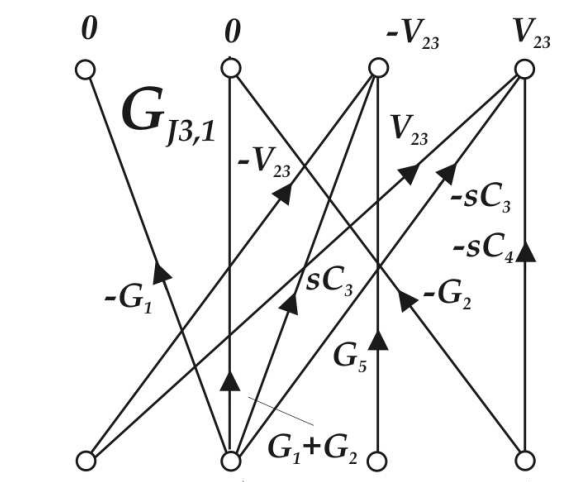

$\partial V_{1} / \partial\left(s C_{3}\right)$

$\partial V_{4} / \partial\left(s C_{3}\right)$

$\partial V_{23} / \partial\left(s C_{3}\right)$

$\partial V_{5} / \partial\left(s C_{3}\right)$

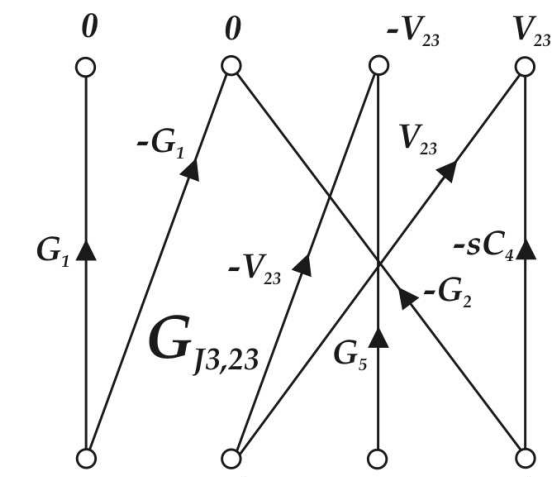

$\partial V_{1} / \partial\left(s C_{3}\right)$

$\partial V_{4} / \partial\left(s C_{3}\right)$

$\partial V_{23} / \partial\left(s C_{3}\right) \quad \partial V_{5} / \partial\left(s C_{3}\right)$
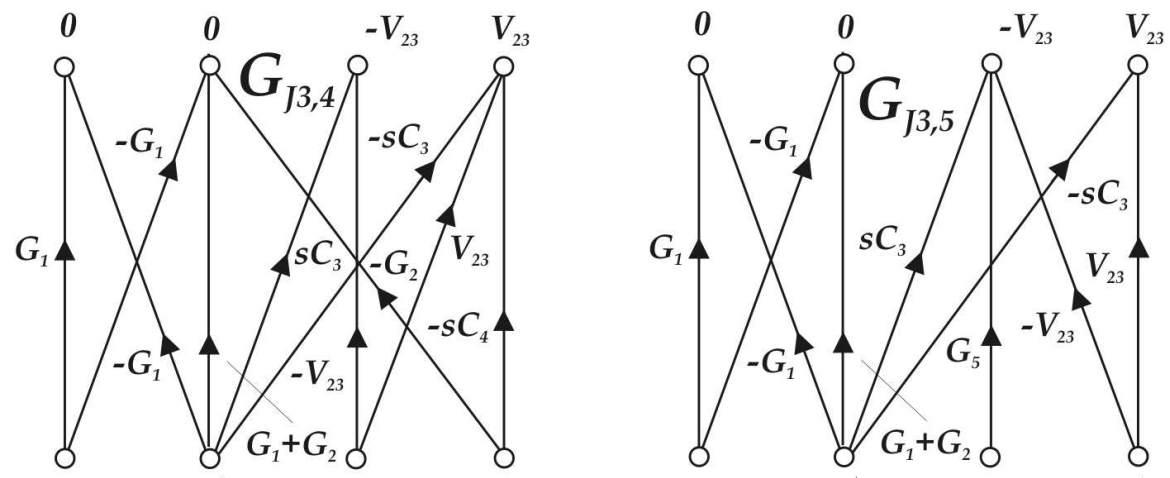

$\partial V_{1} / \partial\left(s C_{3}\right)$

$\partial V_{4} / \partial\left(s C_{3}\right)$

$\partial V_{1} / \partial\left(s C_{3}\right)$

$\partial V_{4} / \partial\left(s C_{3}\right)$

$\partial V_{23} / \partial\left(s C_{3}\right)$

$\partial V_{5} / \partial\left(s C_{3}\right)$

Fig. 9. CM Graphs $G_{J 3,1}, G_{J 3,23,} G_{J 3,4}$ and $G_{J 3,5}$

$\partial V_{23} / \partial\left(s C_{3}\right)$

$\partial V_{5} / \partial\left(s C_{3}\right)$ 


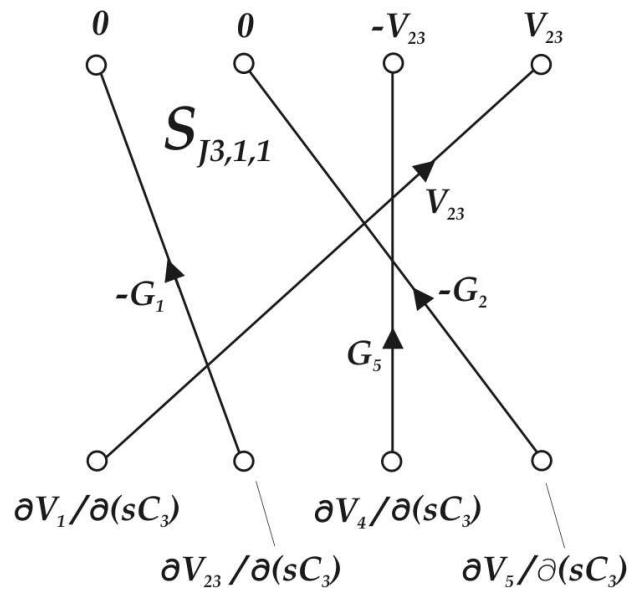

Fig. 10. Separation $S_{J 3,1,1}$ of CM Graph $G_{J 3,1}$

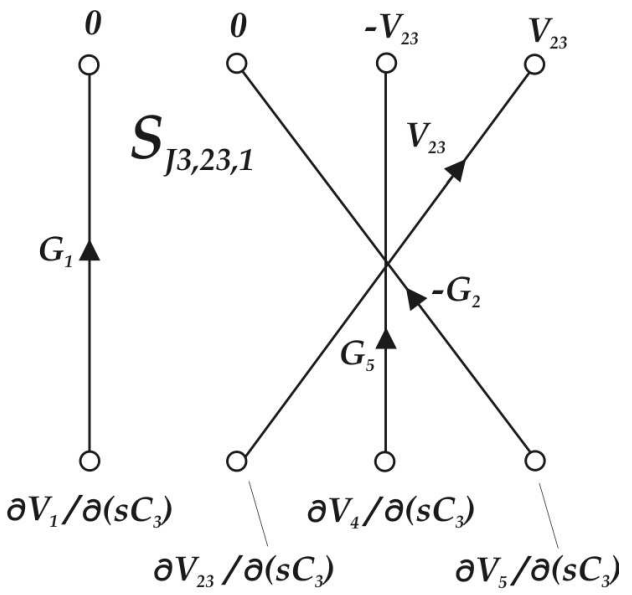

Fig. 11. Separation $S_{J 3,23,1}$ of CM Graph $G_{J 3,23}$ 

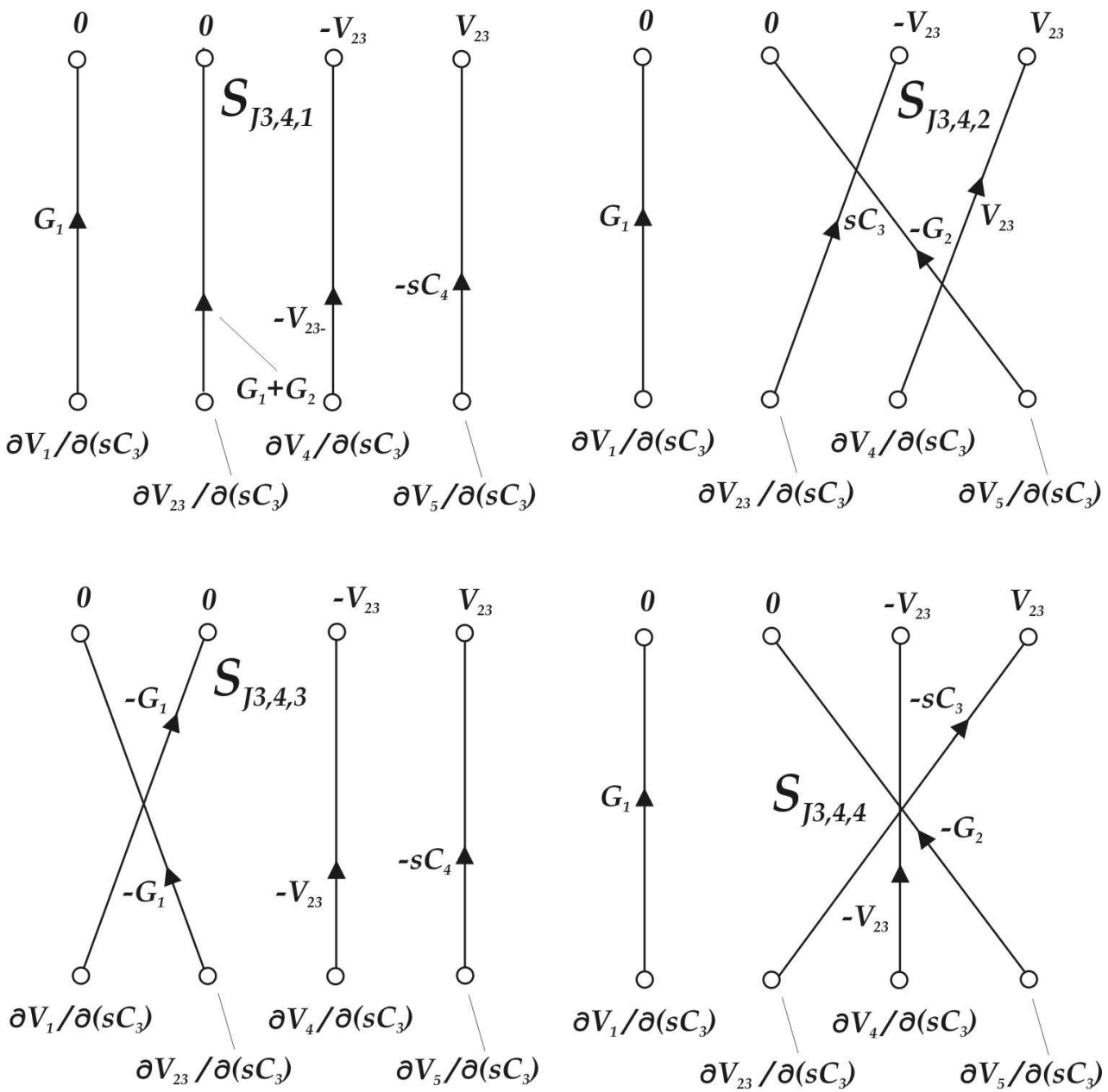

Fig. 12. Separations $S_{J 3,4,1}, S_{J 3,4,2}, S_{J 3,4,3}$ and $S_{J 3,4,4}$ of CM Graph $G_{J 3,4}$ 


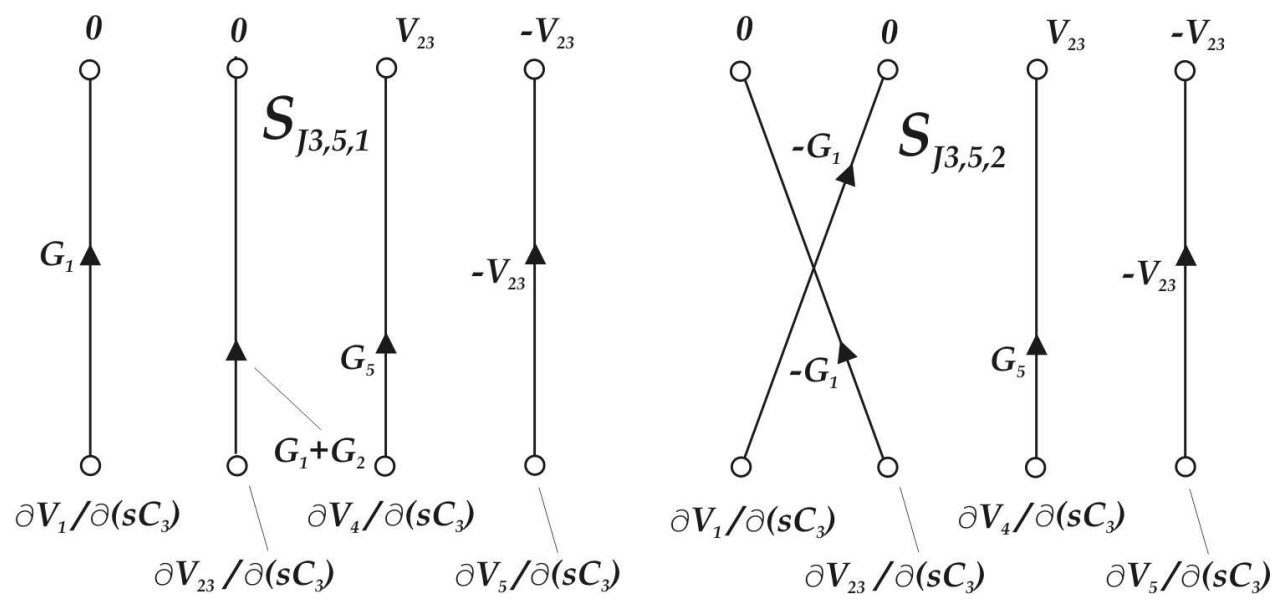

Fig. 13. Separations $S_{J 3,5,1}$ and $S_{J 3,5,2}$ of CM Graph $G_{J 3,5}$

From Fig. $9 \div$ Fig. 13 it follows

$$
\left.\begin{array}{l}
S P_{J 3,1,1}=G_{1} G_{2} G_{5} V_{23} ; N_{a, J 3,1,1}=4 ; N s, J 3,1,1=0 ; \\
S P_{J 3,23,1}=-G_{1} G_{2} G_{5} V_{23} ; N_{a, J 3,23,1}=4 ; N s, J 3,23,1=1 ; \\
S P_{J 3,4,1}=G_{1}\left(G_{1}+G_{2}\right) s C_{4} V_{23} ; N_{a, J 3,4,1}=4 ; N s, J 3,4,1=0 ; \\
S P_{J 3,4,2}=-G_{1} G_{2} S C_{3} V_{23} ; N_{a, J 3,4,2}=4 ; N_{s, J 3,4,2}=0 ; \\
S P_{J 3,4,3}=G_{1}^{2} s C_{4} V_{23} ; N_{a, J 3,4,3}=2 ; N_{s, J 3,4,3}=1 ; \\
S P_{J 3,4,4}=-G_{1} G_{2} S C_{3} V_{23} ; N_{a, J 3,4,4}=2 ; N_{s, J 3,4,4}=1 ; \\
S P_{J 3,5,1}=G_{1}\left(G_{1}+G_{2}\right) C_{5} V_{23} ; N_{a, J 3,5,1}=4 ; N_{s, J 3,5,1}=0 ; \\
S P_{J 3,5,2}=G_{1}^{2} G_{5} V_{23} ; N_{a, J 3,5,2}=2 ; N_{s, J 3,5,2}=1 ;
\end{array}\right\}
$$

Than by substituting (35) in (36) and by taking into consideration (33) from (4) and (5) we obtain the vector $\mathbf{J}_{3}$ :

$$
\mathbf{J}_{3}=\left[\begin{array}{llll}
\frac{J_{1} C_{4}}{G_{2} s\left(C_{3}+C_{4}\right)^{2}} & \frac{J_{1} C_{4}}{G_{2} s\left(C_{3}+C_{4}\right)^{2}} & \frac{J_{1} C_{4}^{2}}{G_{2} G_{5}\left(C_{3}+C_{4}\right)^{2}} & \frac{J_{1} C_{4}}{G_{2} s\left(C_{3}+C_{4}\right)^{2}}
\end{array}\right]_{t}
$$

\section{Hessian matrix determination}

In many practical cases it is necessary and useful to find not only the first-order derivatives of a network function or variable (for example voltage $V_{w}$ ) among $n$ variables with respect to some parameter (for example $y_{s}$ ) but their second-order derivatives with respect to the same or to an other parameter (for example $y_{t}$ ), too.

The matrix formed from all possible second-order derivatives of $V_{w}$ with respect to the simultaneous changes of two parameters 


$$
\mathbf{H}_{w}=\left[\begin{array}{cccc}
\frac{\partial^{2} V_{w}}{\partial y_{1}^{2}} & \frac{\partial^{2} V_{w}}{\partial y_{1} \partial y_{2}} & \cdots & \frac{\partial^{2} V_{w}}{\partial y_{1} \partial y_{n}} \\
\frac{\partial^{2} V_{w}}{\partial y_{2} \partial y_{1}} & \frac{\partial^{2} V_{w}}{\partial y_{2}^{2}} & \cdots & \frac{\partial^{2} V_{w}}{\partial y_{2} \partial y_{n}} \\
\cdot & \cdot & \cdot & \cdot \\
\frac{\partial^{2} V_{w}}{\partial y_{n} \partial y_{1}} & \frac{\partial^{2} V_{w}}{\partial y_{n} \partial y_{2}} & \cdots & \frac{\partial^{2} V_{w}}{\partial y_{n}{ }^{2}}
\end{array}\right]
$$

is the Hessian matrix or briefly Hessian (Korn \& Korn, 1968, Wilde, 1978). Obviously for a network one exists a variety of Hessian matrices - every one matrix corresponds to a definite network function or variable.

The results obtained in section 3. can be applied to the derivation of a Hessian matrix as it will be explained below. By differentiating the vector $\mathbf{J}_{s}$ in (27) with respect to the admittance $y_{t}$ one obtains

$$
\left.\begin{array}{l}
\frac{\partial \mathbf{J}_{s}}{\partial y_{t}}=\frac{\partial^{2} \mathbf{V}}{\partial y_{s} \partial y_{t}}=-\left[\frac{\partial \mathbf{Y}^{-1}}{\partial y_{t}} \mathbf{K}_{s} \mathbf{Y}^{-1}+\mathbf{Y}^{-1} \frac{\partial}{\partial y_{t}}\left(\mathbf{K}_{s} \mathbf{Y}^{-1}\right)\right] \mathbf{I} ; \\
s, t \in\{1,2, \ldots, n\} .
\end{array}\right\}
$$

Because the elements in $\mathbf{Y}$ depend linearly on the network element admittances and their derivatives with respect to the parameter $y_{s}$ equal $1,-1$ or 0 it holds

$$
\frac{\partial \mathbf{K}_{s}}{\partial y_{t}}=0 ; \forall \mathrm{s} ; \frac{\partial \mathbf{Y}}{\partial y_{t}}=\mathbf{K}_{t}
$$

and from (40) it follows

$$
\left.\begin{array}{l}
\frac{\partial^{2} \mathbf{V}}{\partial y_{s} \partial y_{t}}=\mathbf{Y}^{-1}\left(\mathbf{K}_{t} \mathbf{Y}^{-1} \mathbf{K}_{s}+\mathbf{K}_{s} \mathbf{Y}^{-1} \mathbf{K}_{t}\right) \mathbf{Y}^{-1} \mathbf{I}= \\
=\mathbf{Y}^{-1} \mathbf{K}_{s t} \mathbf{V}=\mathbf{Y}^{-1} \mathbf{V}_{s t} ; \\
\mathbf{K}_{s t}=\mathbf{K}_{t} \mathbf{Y}^{-1} \mathbf{K}_{s}+\mathbf{K}_{s} \mathbf{Y}^{-1} \mathbf{K}_{t} ; \\
\mathbf{V}_{s t}=\mathbf{K}_{s t} \mathbf{V}
\end{array}\right\}
$$

The last result compared with the formulae (27) and (28) shows that we can find the vector $\partial^{2} \mathbf{V} / \partial y_{s} \partial y_{t}$ in principle by using the same approach as for $\partial \mathbf{V} / \partial y_{s}$ in section 3 .

However here we must pay attention to the obtaining of the matrix $\mathbf{K}_{s t}$ : In the common case the matrices $\mathbf{K}_{s}$ and $\mathbf{K}_{t}$ contain more than one nonzero element (1 or -1$)$. Hence we can expressed each of them as a sum of no more then four addends

$$
\mathbf{K}_{s}=\sum_{a} \mathbf{K}_{s, a} ; \mathbf{K}_{t}=\sum_{b} \mathbf{K}_{t, b} ; a, b \leq 4
$$


where each of the matrices $\mathbf{K}_{s, a}$ and $\mathbf{K}_{t, b}$ has only one nonzero element. Then as a result the expression of $\mathbf{K}_{s t}$ in (42) is a sum of products of the kind

$$
\mathbf{K}_{s, a} \mathbf{Y}^{-1} \mathbf{K}_{t, b} \text { and } \mathbf{K}_{t, b} \mathbf{Y}^{-1} \mathbf{K}_{s, a} ; \forall a, b \text {. }
$$

The products in (44) are square matrices with only one nonzero element which is a definite element of $\mathbf{Y}^{-1}$. Let, for example, the nonzero element for the left-side matrix in (44) is on $i$-th row and on $j$-th column and the similar element for the right-side matrix is on $k$-th row and on $l$-th column. Then it is easy to see that the corresponding product in (44) contains the element $\pm z_{u, v} ; u, v \in\{1,2, \ldots, n\}$ on $i$-th row and on $l$-th column, where $z_{u, v}$ is an element of $\mathbf{Y}^{-1}$. The upper (lower) sign of this element holds for equal (non equal) signs of nonzero elements of $\mathbf{K}_{s, a}$ and $\mathbf{K}_{t, b}$ in (44), respectively.

The matrix $\mathbf{Y}^{-1}$ can be evaluated by using an auxiliary $\mathrm{CM}$ graph $G_{0}$ too. For this purpose let us consider the equation

$$
\mathbf{X}=\mathbf{Y}^{-1} \mathbf{E},
$$

where

$$
\left.\mathbf{Y}^{-1}=\left[\begin{array}{cccc}
z_{11} & z_{12} & \cdot & z_{1 n} \\
z_{21} & z_{22} & \cdot & z_{2 n} \\
\cdot & \cdot & \cdot & \cdot \\
z_{n 1} & z_{n 2} & \cdot & z_{n n}
\end{array}\right] ; \mathbf{X}=\left[\begin{array}{llll}
x_{1} & x_{2} & \cdot & x_{n}
\end{array}\right]_{t} ; \mathbf{E}=\left[\begin{array}{llll}
e_{1} & e_{2} & \cdot & e_{n}
\end{array}\right]_{t} \cdot\right\}
$$

After multiplying in (45) for $\mathbf{X}$ one follows

$$
\mathbf{X}=\left[\begin{array}{c}
z_{11} e_{1}+z_{12} e_{2}+\ldots+z_{1 n} e_{n} \\
z_{21} e_{1}+z_{22} e_{2}+\ldots+z_{2 n} e_{n} \\
\cdot \\
z_{n 1} e_{1}+z_{n 2} e_{2}+\ldots+z_{n n} e_{n}
\end{array}\right]
$$

This means that if the CM graph $G_{0}$ corresponds to (45) the multipliers of $e_{1}, e_{2}, \ldots, e_{n}$ for every element of $\mathbf{X}$ are elements of $\mathbf{Y}^{-1}$. Note that in real cases a limited number of the elements of $\mathbf{Y}^{-1}$ are necessary only. Hence for determination of an element of the Hessian matrix $\mathbf{H}_{s t}$ we can form the following:

\section{Rule 3:}

i. Draw the $\mathrm{CM}$ graph $G_{p}$ of the nullor network under consideration;

ii. Transform the graph $G_{p}$ into the graph $G$, according to the Rule 1 in section 2 . and compose the vectors $\mathbf{V}$ and $\mathbf{I}$;

iii. Determine the vector $\mathbf{V}$ from $G$;

iv. Write the matrices $\mathbf{K}_{s}$ and $\mathbf{K}_{t}$;

v. Determine the matrix $\mathbf{Y}^{-1}$ by using the auxiliary $C M$ graph $G_{0}$;

vi. Determine the matrix $\mathbf{K}_{\text {st }}$;

vii. Determine the matrix $\mathbf{V}_{s t}$;

viii. Draw a $C M$ graph $\boldsymbol{G}_{s t}$ in accordance with $\mathbf{V}_{s t}$;

ix. Determine the elements of the vector $\partial^{2} \mathbf{V} / \partial y_{s} \partial y_{t}$ from $\boldsymbol{G}_{s t}$. 
Note that by following the above sequence we obtain $2 n$ elements of $n$ Hessian matrices simultaneously, because $\partial^{2} \mathbf{V} / \partial y_{s} \partial y_{t}=\partial^{2} \mathbf{V} / \partial y_{t} \partial y_{s}-$ Fig. 14.

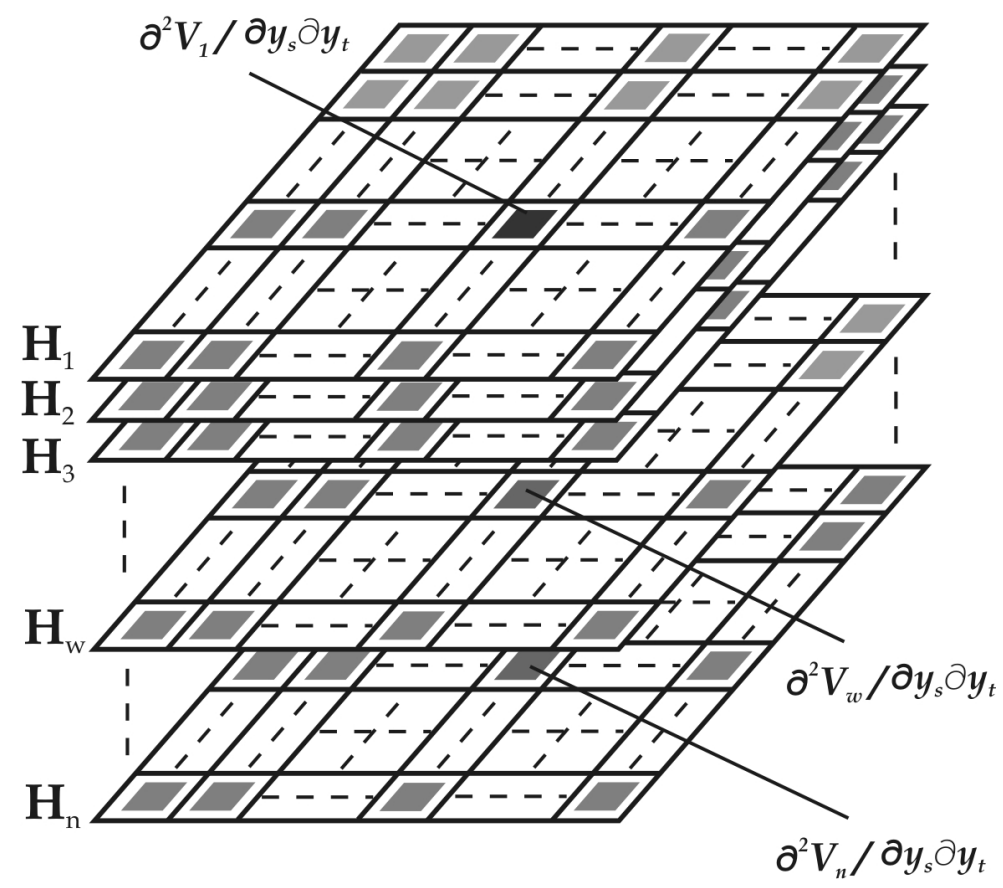

Fig. 14. A Set of $n$ Hessian Matrices

\section{Example B}

Suppose that we want to determine the vector $\partial^{2} \mathbf{V} / \partial\left(s C_{3}\right) \partial\left(s C_{4}\right)$ for the network $N$ in Fig.2. Because the items i, ii and iii of the Rule 3 were fulfilled in the Example A we have to continue further: Here the matrices $\mathbf{K}_{3}$ and $\mathbf{K}_{4}$ are

$$
\left.\begin{array}{c}
\mathbf{K}_{31}=\left[\begin{array}{llll}
0 & 0 & 0 & 0 \\
0 & 0 & 0 & 0 \\
0 & 1 & 0 & 0 \\
0 & 0 & 0 & 0
\end{array}\right] ; \mathbf{K}_{32}=\left[\begin{array}{cccc}
0 & 0 & 0 & 0 \\
0 & 0 & 0 & 0 \\
0 & 0 & 0 & 0 \\
0 & -1 & 0 & 0
\end{array}\right] ; \\
\mathbf{K}_{3}=\mathbf{K}_{31}+\mathbf{K}_{32} ; \mathbf{K}_{4}=\left[\begin{array}{cccc}
0 & 0 & 0 & 0 \\
0 & 0 & 0 & 0 \\
0 & 0 & 0 & 0 \\
0 & 0 & 0 & -1
\end{array}\right]
\end{array}\right\}
$$

and from $(42) \div(46)$ it follows 


$$
\mathbf{K}_{34}=\left[\begin{array}{cccc}
0 & 0 & 0 & 0 \\
0 & 0 & 0 & 0 \\
0 & 0 & 0 & -z_{24} \\
0 & -z_{43}+z_{44} & 0 & z_{24}
\end{array}\right] .
$$

We can find the nonzero elements of $\mathbf{K}_{34}$ by using the auxiliary $\mathrm{CM}$ graph $\boldsymbol{G}_{0}$ drawn in Fig. 15. By comparing (47) with (49) one settles we need only these addends of elements $x_{2}$ and $x_{4}$ in (47) that content the quantities $e_{4}$ and $e_{3}, e_{4}$, respectively. According to the Chan-Mai procedure we draw the graphs $G_{0,2}$ and $G_{0,4}$ - Fig. 16 and Fig. 17.

$$
z_{24}=z_{44}=-\frac{1}{s\left(C_{3}+C_{4}\right)} ; z_{43}=0
$$

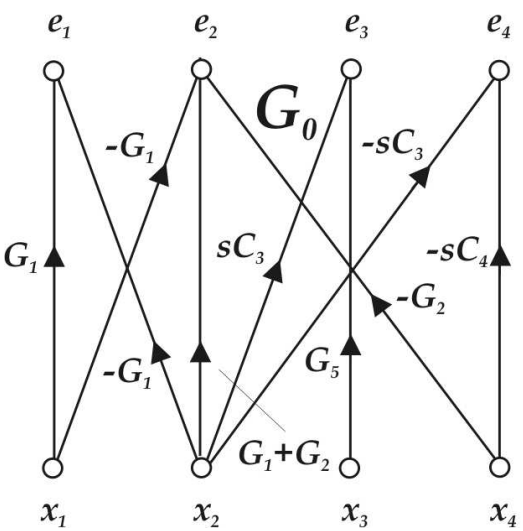

Fig. 15. The auxiliary $\mathrm{CM}$ graph $G_{0}$

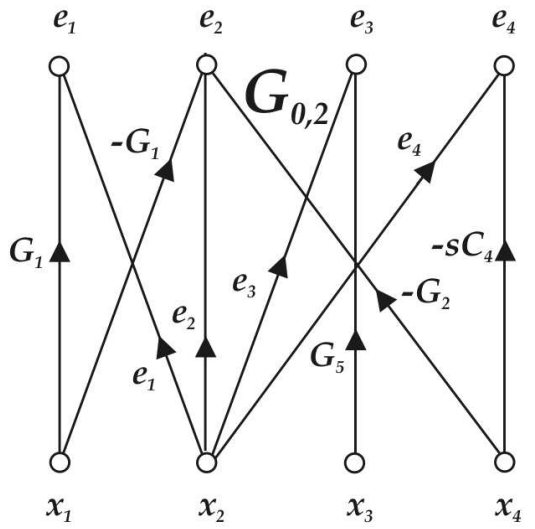

Fig. 16. The CM graph $G_{0,2}$ 


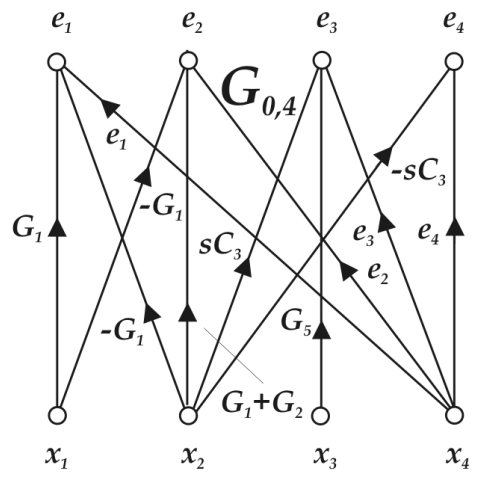

Fig. 17. The CM graph $G_{0,4}$

From Fig. $18 \div$ Fig. 19 we obtain the products

$$
\left.\begin{array}{l}
S P_{0,2,1}=-G_{1} s C_{4} G_{5} e_{2} ; N_{a, 0,2,1}=4 ; N_{s, 0,2,1}=0 ; \\
S P_{0,2,2}=G_{1} s C_{4} G_{5} e_{1} ; N_{a, 0,2,2}=2 ; N_{s, 0,2,2}=1 ; \\
S P_{0,2,3}=-G_{1} G_{2} G_{5} e_{4} ; N_{a, 0,2,3}=2 ; N_{s, 0,2,3}=1 ; \\
S P_{0,4,1}=G_{1}\left(G_{1}+G_{2}\right) G_{5} e_{4} ; N_{a, 0,4,1}=4 ; N_{s, 0,4,1}=0 ; \\
S P_{0,4,2}=G_{1}^{2} G_{5} e_{4} ; N_{a, 0,4,2}=2 ; N_{s, 0,4,2}=1 ; \\
S P_{0,4,3}=-G_{1} s C_{3} G_{5} e_{2} ; N_{a, 0,4,3}=2 ; N_{s, 0,4,3}=1 ;
\end{array}\right\} .
$$

Note that with the exception of the sink and source quantities the graph $G_{0}$ is isomorphic to the graph $G$ in Fig. 4. That is why the expressions (33) remain valid for the denominator in (4) also. Than for the elements of the vector (47) from (51) and (33) it follows:

$$
\left.\begin{array}{l}
x_{2}=\frac{G_{1} s C_{4} G_{5} e_{1}+G_{1} s C_{4} G_{5} e_{2}-G_{1} G_{2} G_{5} e_{4}}{G_{1} G_{2} G_{5} s\left(C_{3}+C_{4}\right)} ; \\
x_{4}=\frac{-G_{1} s C_{3} G_{5} e_{2}-G_{1} G_{2} G_{5} e_{4}}{G_{1} G_{2} G_{5} s\left(C_{3}+C_{4}\right)}
\end{array}\right\}
$$

or taking into consideration (46) and (47)
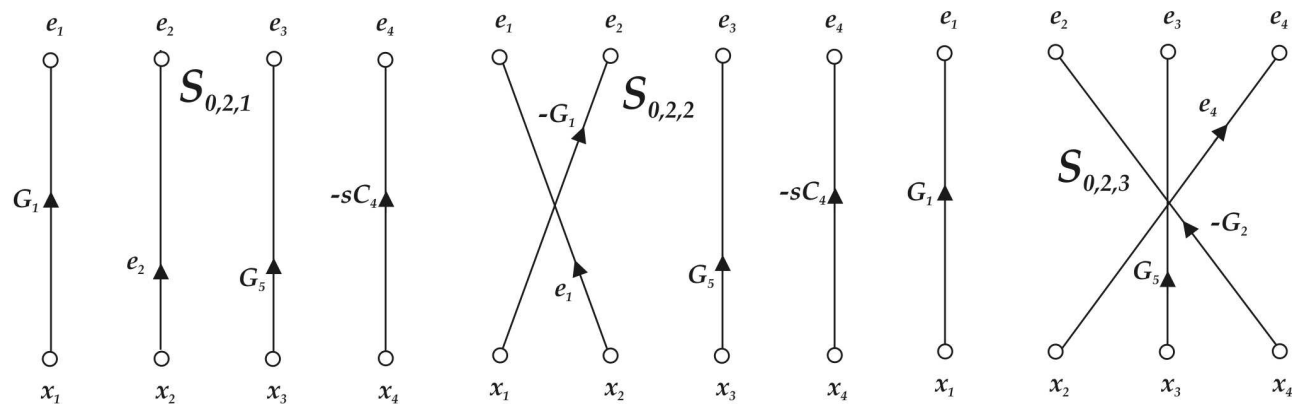

Fig. 18. Separations $S_{0,2,1}, S_{0,2,2}$ and $S_{0,2,3}$ of CM graph $G_{0,2}$ 

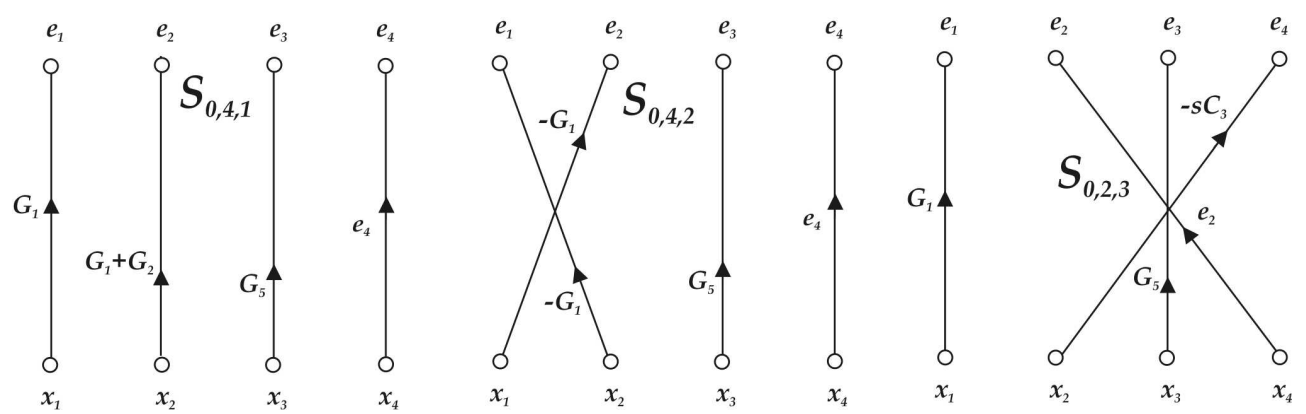

Fig. 19. Separations $S_{0,4,1}, S_{0,4,2}$ and $S_{0,4,3}$ of $C M$ graph $G_{0,4}$

$$
\left.z_{24}=z_{44}=-\frac{1}{s\left(C_{3}+C_{4}\right)} ; z_{43}=0\right\} .
$$

Now we return to (42) and (49) and obtain

$$
\left.\begin{array}{rl}
\left.\mathbf{K}_{34}=\left[\begin{array}{cccc}
0 & 0 & 0 & 0 \\
0 & 0 & 0 & 0 \\
0 & 0 & 0 & \frac{1}{s\left(C_{3}+C_{4}\right)} \\
0 & -\frac{1}{s\left(C_{3}+C_{4}\right)} & 0 & -\frac{1}{s\left(C_{3}+C_{4}\right)}
\end{array}\right] ; \mathbf{V}_{34}=\mathbf{K}_{34} \mathbf{V}=\mathbf{K}_{34}\left[\begin{array}{c}
V_{1} \\
V_{23} \\
V_{4} \\
V_{5}
\end{array}\right]=\left[\begin{array}{c}
0 \\
0 \\
\frac{V_{5}}{s\left(C_{3}+C_{4}\right)} \\
-\frac{V_{23}+V_{5}}{s\left(C_{3}+C_{4}\right)}
\end{array}\right]\right\} . \\
S P_{H, 34,1,1}=-G_{1} G_{2} G_{5} \frac{V_{23}+V_{5}}{s\left(C_{3}+C_{4}\right)} ; N_{a, J 3,1,1}=4 ; N s, J 3,1,1=0 ; \\
S P_{H, 34,23,1}=G_{1} G_{2} G_{5} \frac{V_{23}+V_{5}}{s\left(C_{3}+C_{4}\right)} ; N_{a, J 3,23,1}=4 ; N s, J 3,23,1=1 ; \\
S P_{H, 34,4,1}=-G_{1}\left(G_{1}+G_{2}\right) s C_{4} \frac{V_{5}}{s\left(C_{3}+C_{4}\right)} ; N_{a, J 3,4,1}=4 ; N s, J 3,4,1=0 ; \\
S P_{H, 34,4,2}=G_{1} G_{2} s C_{3} \frac{V_{23}+V_{5}}{s\left(C_{3}+C_{4}\right)} ; N_{a, J 3,4,2}=4 ; N_{s, J 3,4,2}=0 ; \\
S P_{H, 34,4,3}=-G_{1}^{2} s C_{4} \frac{V_{5}}{s\left(C_{3}+C_{4}\right)} ; N_{a, J 3,4,3}=2 ; N_{s, J 3,4,3}=1 ; \\
S P_{H, 34,4,4}=G_{1} G_{2} s C_{3} \frac{V_{5}}{s\left(C_{3}+C_{4}\right)} ; N_{a, J 3,4,4}=2 ; N_{s, J 3,4,4}=1 ; \\
S P_{H, 34,5,1}=-G_{1}\left(G_{1}+G_{2}\right) C_{5} \frac{V_{23}+V_{5}}{s\left(C_{3}+C_{4}\right)} ; N_{a, J 3,5,1}=4 ; N_{s, J 3,5,1}=0 ; \\
S P_{H, 34,5,2}=-G_{1}^{2} G_{5} \frac{V_{23}+V_{5}}{s\left(C_{3}+C_{4}\right)} ; N_{a, J 3,5,2}=2 ; N_{s, J 3,5,2}=1 ;
\end{array}\right\} .
$$


In order to determine the second derivatives of the vector $\partial^{2} \mathbf{V} / \partial\left(s C_{3}\right) \partial\left(s C_{4}\right)$ and having in mind (42) one draws the $\mathrm{CM}$ graph $G_{H, 34}$ shown in Fig. 20. In the case we have a simplification of the analysis on the base of the graph $G_{H, 34}$ because the substantial difference between $G_{H, 34}$ and $G_{J 3}$ consists in the sink and source vertex signal expressions instead of $-V_{23}$ and $V_{23}$ in $G_{J 3}$ the corresponding signals in $G_{H, 34}$ are $V_{5} / s\left(C_{3}+C_{4}\right)$ and $\left(V_{23}+V_{5}\right) / s\left(C_{3}+C_{4}\right)$. Owing to this peculiarity further we use directly (37) after substituting sink vertex signals, namely:

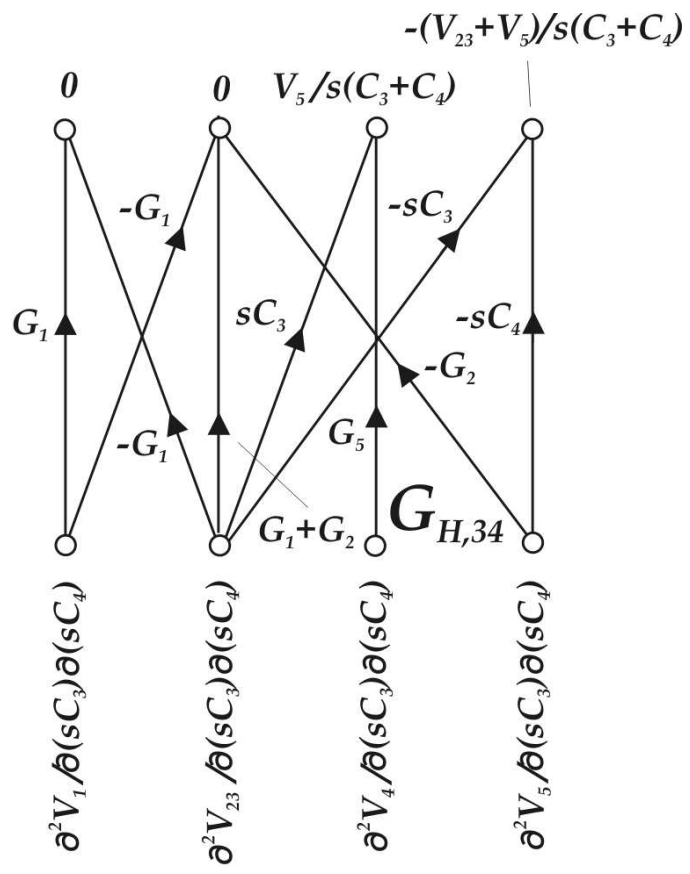

Fig. 20. CM Graph $G_{H, 34}$

The voltage $V_{5}$ can be find similarly to $V_{23}$ from $C M$ graph $G$ and it is:

$$
V_{5}=-\frac{C_{3} J_{1}}{s\left(C_{3}+C_{4}\right)}
$$

Then by using (33), (35), (55) and (56) one obtains the vector

$$
\frac{\partial^{2} \mathbf{V}}{\partial\left(s C_{3}\right) \partial\left(s C_{4}\right)}=\left[\begin{array}{llll}
\frac{J_{1}\left(C_{4}-C_{3}\right)}{G_{2} s^{2}\left(C_{3}+C_{4}\right)^{3}} & \frac{J_{1}\left(C_{4}-C_{3}\right)}{G_{2} s^{2}\left(C_{3}+C_{4}\right)^{3}} & -2 \frac{J_{1} C_{3} C_{4}}{s G_{2} G_{5}\left(C_{3}+C_{4}\right)^{3}} & \frac{J_{1}\left(C_{4}-C_{3}\right)}{G_{2} s^{2}\left(C_{3}+C_{4}\right)^{3}}
\end{array}\right]_{t},
$$

Its elements are a part of elements in the Hessian matrices $\mathbf{H}_{1}, \mathbf{H}_{23}, \mathbf{H}_{4}$ and $\mathbf{H}_{5}$ with respect to the admittances $s C_{3}$ and $s C_{4}$. 


\section{First and second-order quadratic sensitivity sums}

The sensitivity is an important parameter for the evaluation of practical suitability of electrical networks. For this purpose usually one uses the first-order sensitivity and the second-order sensitivity, defined by the well known formulae (Cederbaum, 1984; Chua \& Lin, 1975)

$$
S_{x}^{F}=\frac{\partial F}{\partial x} \cdot \frac{x}{F}
$$

and

$$
S_{x, y}^{F}=\frac{\partial^{2} F}{\partial x \partial y} \cdot \frac{x y}{F},
$$

respectively and where $F$ is a network function or variable and $x, y$ are changeable network element parameters.

Obviously, the derivatives in these expressions can be determined according to the above described method based on Chan-Mai signal-flow graphs. Besides very often we are interested in a global index as a quadratic sum of sensitivities (first- or second-order):

$$
\sum_{i}\left(S_{x}^{F_{i}}\right)^{2}=\sum_{i}\left(\frac{\partial F_{i}}{\partial x} \cdot \frac{x}{F_{i}}\right)^{2}
$$

and

$$
\sum_{i}\left(S_{x, y}^{F_{i}}\right)^{2}=\sum_{i}\left(\frac{\partial^{2} F_{i}}{\partial x \partial y} \cdot \frac{x y}{F_{i}}\right)^{2}
$$

where $i \in\{1,2, \ldots, n\}$.

Without loss of generality further we assume that the functions $F_{i}$ are the elements of the voltage vector $\mathbf{V}$. Then the sum (60) can be derived with the help of the expressions of the corresponding Jacobian matrix subvectors $\mathbf{J}_{i}$ and of the voltage vector $\mathbf{V}$ :

$$
\left.\begin{array}{l}
\sum_{i}\left(S_{x}^{V_{i}}\right)^{2}=x^{2} \mathbf{J}_{i, t}\left(\mathbf{M}^{-1}\right)^{2} \mathbf{J}_{i} ; \\
\mathbf{M}=\operatorname{diag}\left\{V_{1}, V_{2}, \ldots, V_{i}, \ldots, V_{n}\right\}
\end{array}\right\} .
$$

If from the elements of the Hessian matrices $\mathbf{H}_{i}$ one forms the vector

$$
\mathbf{h}_{x y}=\left[\begin{array}{llllll}
h_{1, x y} & h_{2, x y} & \ldots & h_{i, x y} & \ldots & h_{n, x y}
\end{array}\right]_{t} ; h_{i, x y}=\frac{\partial^{2} V_{i}}{\partial x \partial y}
$$


the sum (61) can be rewritten as

$$
\sum_{i}\left(S_{x y}^{V_{i}}\right)^{2}=x^{2} y^{2} \mathbf{h}_{i, t}\left(\mathbf{M}^{-1}\right)^{2} \mathbf{h}_{i}
$$

\section{Conclusions}

A topological method for obtaining the Jacobian and Hessian matrices and their use for quadratic first- or second-order sensitivity sums calculation of active networks is presented. It is based on the replacement of the investigated network $N$ by using a nullor equivalent circuit and on the representation of the circuit passive part $N_{p}$ by a Chan-Mai signal-flow graph $G_{p}$. The Jacobian and the Hessian matrix elements of the nullor network can be obtained by means of the some dependent variables of some Chan-Mai graphs derived from $G$. The substantial advantage of the method consists in the use mainly of isomorphic graphs. Two examples illustrate the proposed method.

\section{Acknowledgement}

The author would like to thank Higher School of Transport "Todor Kableshkov", Sofia, Bulgaria for the financial support for the publishing this work.

\section{References}

Cederbaum, I. (1984). "Some Applications of Graph Theory to Network Analysis and Synthesis", IEEE Tr. on Circuits and Systems, vol. CAS-31, 1, 1984, pp. 64-68

Chan, S.P., Mai, H.N. (1967). "A Flow-Graph Method for the Analysis of Linear Systems", IEEE Tr. on Circuit Theory, 9, 1967, pp. 350-354

Chua, L.O., Lin, P.-M. (1975). “Computer-Aided Analysis of Electronic Circuits”, Prentice-Hall Inc. Englewood Clifs, New Jersey, 1975

Davies, A. C. (1966). "Matrix Analysis of Network Containing Nullators and Norators", Electronics Letters, vol. 2, ,2, 1966, pp. $48-49$

Donevsky, B.D., Nenov,G.A. (1979). "Application of Graphs for the Analysis and Synthesis of Electronic Circuits”, “Technica”, Sofia, 1979 (in Bulgarian)

Korn, G., A., Korn, T.M. (1968). “Mathematical Handbook”, Mc Graw-Hill Co. New York. 1968

Nenov, G. A. (2004). "Evaluation of Nullor Nertwork Jacobian Matrix by using Chan-Mai Signal-Flow Graphs", Proceedings of the SMACD'04, Wroclaw, 2004, Poland, pp. 7982

Wilde, D.J. (1978). “Globally Optimal Design”, John Wiley \& Sons, New York, 1978 


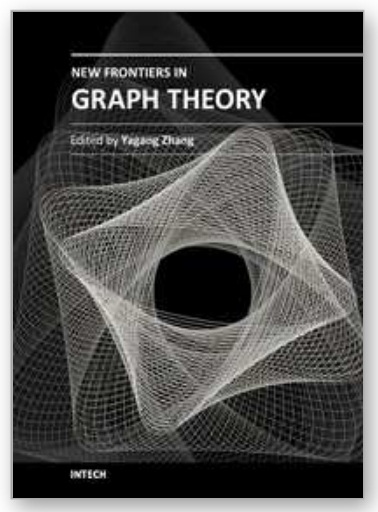

\author{
New Frontiers in Graph Theory \\ Edited by Dr. Yagang Zhang
}

ISBN 978-953-51-0115-4

Hard cover, 526 pages

Publisher InTech

Published online 02, March, 2012

Published in print edition March, 2012

Nowadays, graph theory is an important analysis tool in mathematics and computer science. Because of the inherent simplicity of graph theory, it can be used to model many different physical and abstract systems such as transportation and communication networks, models for business administration, political science, and psychology and so on. The purpose of this book is not only to present the latest state and development tendencies of graph theory, but to bring the reader far enough along the way to enable him to embark on the research problems of his own. Taking into account the large amount of knowledge about graph theory and practice presented in the book, it has two major parts: theoretical researches and applications. The book is also intended for both graduate and postgraduate students in fields such as mathematics, computer science, system sciences, biology, engineering, cybernetics, and social sciences, and as a reference for software professionals and practitioners.

\title{
How to reference
}

In order to correctly reference this scholarly work, feel free to copy and paste the following:

Georgi A. Nenov (2012). Symbolic Determination of Jacobian and Hessian Matrices and Sensitivities of Active Linear Networks by Using Chan-Mai Signal-Flow Graphs, New Frontiers in Graph Theory, Dr. Yagang Zhang (Ed.), ISBN: 978-953-51-0115-4, InTech, Available from: http://www.intechopen.com/books/new-frontiers-ingraph-theory/symbolic-determination-of-jacobian-and-hessian-matrices-and-sensitivities-of-active-linearnetworks-

\section{INTECH}

open science | open minds

\section{InTech Europe}

University Campus STeP Ri

Slavka Krautzeka 83/A

51000 Rijeka, Croatia

Phone: +385 (51) 770447

Fax: +385 (51) 686166

www.intechopen.com

\section{InTech China}

Unit 405, Office Block, Hotel Equatorial Shanghai

No.65, Yan An Road (West), Shanghai, 200040, China

中国上海市延安西路65号上海国际贵都大饭店办公楼405单元

Phone: +86-21-62489820

Fax: $+86-21-62489821$ 
(C) 2012 The Author(s). Licensee IntechOpen. This is an open access article distributed under the terms of the Creative Commons Attribution 3.0 License, which permits unrestricted use, distribution, and reproduction in any medium, provided the original work is properly cited. 